\title{
ACUTE CHANGES IN ENERGY EXPENDITURE AND HEART RATE VARIABILITY DURING AND RIGHT AFTER ONE HOUR OF HATHA YOGA PRACTICE
}

\author{
MEHMET EMIN TUNA', DICLE ARAS², NEVAL ARAS³, MEHMET ALI ÖZÇELIK4, \\ ABDURRAHMAN AKTOP 4 \\ Independent researcher \\ 2Ankara University, Faculty of Sport Sciences, Department of Coaching Education, Turkey \\ 3 Sensing Body Academy, Turkey \\ ${ }^{4} A k d e n i z$ University, Faculty of Sport Sciences, Turkey
}

\begin{abstract}
Mailing address: Dicle Aras, Ankara University, Faculty of Sport Sciences, 50. Yil Yerleskesi Street, 06830 Golbasi, Ankara, Turkey, tel.: +90 (312) 600 0100-1644, e-mail: daras@ankara.edu.tr
\end{abstract}

\begin{abstract}
Introduction. This study purposed to examine energy expenditure (EE) of one-hour hatha yoga (HY) practice and to compare changes in heart rate variability (HRV) during and right after HY practice. Material and methods. A total of ten experienced female HY practitioners participated in the study voluntarily. Daily EE on a weekday and during a one-hour HY session was measured using a metabolic holter. The ECG holter was applied to record HRV variables 5 minutes before, during, 5 minutes after and 10 minutes after the one-hour HY session. The HY session included HY asanas for the first 55 minutes and meditation during the last 5 minutes. Results. Participants showed $2201.40 \mathrm{kcal}$ of total EE, and $421.70 \mathrm{kcal}$ of active EE during the weekday. Active EE was $109.70 \mathrm{kcal}$ ( $26 \%$ of daily active EE) during the one-hour HY session, and the mean MET was recorded as 2.57. HRV measurements showed crucial changes. Increased sympathetic activity observed in SDNN, RMSSD, NN50, pNN50, LF/HF, and HF did not reach the initial level after 15 minutes of total recovery time. Conclusions. One hour of HY practice provided moderate EE. Moreover, 10 minutes of supine rest in addition to 5 minutes of the meditation phase of HY practice is not seen as completely effective in improving parasympathetic activity and returning the practitioners to their initial level. Extending the meditation phase at the end of the HY session by more than 10 minutes and measuring the recovery process of HRV could add more detailed results to the literature.
\end{abstract}

Key words: energy expenditure, hatha yoga, heart rate variability, recovery

\section{Introduction}

Yoga is considered as an ancient Indian form consisting of philosophy and practice [1]. Yoga has been performed by both healthy individuals $[2,3]$ and patients with different chronic diseases $[4,5,6]$ and its positive effects in both groups have been reported in the previous studies [2, 7, 8, 9]. According to Papp et al. [10], yoga activities generally provide light-to-moderate physical load. However, some yoga styles such as hatha yoga, involving static muscle contraction with longer durations, have more intense exercises. One of the most practiced types of yoga, i.e. hatha yoga (HY), has been known as a whole, which includes breathing exercises (pranayama), intense physical postures (asanas), and meditation practices [6, 7, 11]. Positive influences of slow, controlled breathing exercise on heart rate variability (HRV) and parasympathetic nervous system have been indicated in a number of studies [12, 13, 14, 15]. The physical activity part (asanas) of HY is considered as high intensity yoga [10]. Positive effects of yoga exercise on muscle strength, muscle endurance and flexibility were reported in the previous studies [16, 17]. It is thought that meditative practices provide mindfulness which minimizes negative effects of chronic pain by reducing punitive self-judgment $[18,19]$ as well as levels of anxiety and depression $[9,20]$ in daily life.

Energy expenditure (EE) is the metabolic equivalent of physical activity, and is determined to provide the caloric balance. Therefore, it is substantial to examine the energy cost of HY in which the participation increases day by day, and compare it with the suggested physical activity level to maintain and/or improve health [21]. In addition to the EE measurements, the observation of the changes of HRV during a HY session could give more detailed information due to the structure of HRV considered as a non-invasive method to evaluate autonomic heart functions in sport sciences [22].

Seeing that yoga has become a popular activity [3], a number of studies have investigated the beneficial effects of yoga practices in recent years $[6,10,23,24]$. However, the present study is the first which aimed to measure the acute changes in energy expenditure and HRV during one hour of HY practice concurrently. Thus, the present study focused on comparing energy cost with the daily energy expenditure, and investigating the acute alterations in HRV parameters in order to understand the functions of sympathetic and parasympathetic systems during one hour of HY practice.

\section{Material and Methods}

A total of 10 healthy female hatha yoga practitioners, with at least two years of hatha yoga experience, participated in the study voluntarily. Individuals who performed hatha yoga (HY) only up to two days per week, had any pain or used any medicaments were excluded from the study. The mean age of the participants was $34.40 \pm 7.43$ years, body weight $60.53 \pm 4.26 \mathrm{~kg}$, body height $170.40 \pm 3.17 \mathrm{~cm}$ and body mass index was $20.86 \pm$ 1.61. Their estimated maximal HR was $182.92 \pm 5.20 \mathrm{bpm}$, and it was found using Gellish et al.'s formula [25]. 
This study was designed to examine energy expenditure (EE) of one-hour HY, and to compare changes in HRV before, during and right after one hour of HY practice. In order to achieve this aim, a yoga studio was visited twice. After giving information and collecting the required forms, armband metabolic holters were placed to all the subjects to measure their daily energy expenditure during the first visit. The second visit took place in the same studio. Firstly, the data of the daily energy expenditure of the participants were recorded. Subsequently, they were asked to lie down, and the holter ECG as well as the metabolic holters were placed. In both situations, all participants were warned against joining any physical activity program the day before and during the measurement day. They were also asked to stop alcohol and caffeine consumption at least 12 hours prior to the measurements. During the second visit, they were asked to lie down for five minutes without speaking and moving. After that, they started a one-hour yoga practice. After completing the HY practice, they were asked to lie down for another 10 minutes. On the same day, EE was recorded during one hour of HY practice, and the HRV recording was started 5 minutes before, and ended 10 minutes after the HY practice. Due to the fact that some phases, such as luteal, can cause an increase in sympathetic activity, all measurements were performed during the follicular phase of the menstrual cycle [26].

The HY program included yoga postures/poses with synchronized breathing. The subjects were told to synchronize their breathing with each movement during the whole session. Each person participated in the same HY program. It started with a 9-minute sun solution A series, repeating 11 poses for 12 times, and continued with some poses with flow series between them for 46 minutes, and ended with 5 minutes of lying on the back (savasana). The sun solution A series consisted of mountain, upward salute, standing forward bend, standing half forward bend, four-limbed staff, upward facing dog, downward facing dog, standing half forward bend, standing forward bend, upward salute and mountain poses. Warrior II, triangle, wide-legged standing forward bend, half-moon, hand-to-big toe, child, half boat, boat, garland, crane, shoulder stand, hero, locust, bow, head-to-knee, half lord of the fish, wide angle seated forward bend, staff, seated forward bend, upward plank, supine spinal twist and corpse poses were chosen as basic poses. Downward facing dog, standing half forward bend, standing forward bend, upward salute, mountain, upward salute, standing forward bend, standing half forward bend, downward facing dog, plank, four-limbed staff, upward facing and downward facing poses were utilized for the short flow series.

Energy expenditure (EE) was measured using the SenseWear Pro armband metabolic holter (Pittsburgh, PA, USA) twice. For the first time, in order to record the subjects' daily $\mathrm{EE}$, a weekday was chosen. The holter was placed on the triceps muscle of the right arm, and stayed there for 24 hours. All measurements began at $10 \mathrm{am}$, and the participants were told to remove the device only when taking a shower. The second use of the metabolic holter was in the yoga studio, and the device was kept on the right arm's triceps during the whole HY practice. The parameters derived from the software and used in the present study were total energy expenditure (TEE, kcal), active energy expenditure (AEE, kcal), number of steps (NOS), average MET (AMET), sleep duration (SD, min), sleep efficiency (SE, \%), lying down duration (LDD, min), sedentary life duration (SLD, min), total physical activity duration (TPAD, min), average physical activity duration (APAD, min) and vigorous physical activity duration (VPAD, min).
Heart rate variability (HRV) measurements were collected using a 3-ch ECG holter (BORSAM, Shenzhen, China). First, the 5-pin cable was placed and then the participants were asked to lie down for five minutes without speaking and moving. At the end of the five minutes the cardiac functional state was recorded as resting condition (HY5minB). Afterwards, the subjects started a one-hour HY practice (HY). The HY practice consisted of 55 minutes of $\mathrm{HY}$ poses and 5 minutes of meditative relaxation. When the practice was finished, they lied down for another 10 minutes for recovery, and HRV was recorded during the whole HY practice and recovery. The HRV parameters listed in the results were HY5minB, HY, HYend, HY5minA, and HYlOminA. HY5minB is the mean value of the collected data during the 5 minutes of resting time before the HY practice was started. HY is the mean collected during the 55 minutes of HY poses. HYend is the value which was recorded at the end of the one-hour HY practice, including 5 minutes of meditative relaxation. The values shown at HY5minA and HYlOminA marked $5^{\text {th }}$ and $10^{\text {th }}$ minutes of the recovery after the HY practice. The time-domain HRV parameters obtained from the ECG holter were HRave, SDNN, RMSSD, SDSD, NN50, pNN50, TRI, and the frequency-domain parameters were TP, LF/HF, HF, LF, VLF and ULF.

SPSS 20 (SPSS Inc., Chicago, IL, USA) was used for all statistical data analyses in the research. Demographic data for the participants are presented as mean \pm SD for both energy expenditure and HRV values. While showing the amount of the changes in the energy expenditure parameters, percentages were also used. To compare the mean differences in HRV parameters obtained from five separate times, firstly, the distribution of data was analyzed with the Shapiro Wilk test. After confirming the distribution, the differences in HRV parameters were compared using Repeated Measures ANOVA or the Friedman Test. In order to determine which group had different data in the variance analysis in which the Friedman Test was used, the analysis was performed with the Wilcoxon Test. Alpha value was accepted as 0.05 for all statistical analyses.

All the participants were informed about the study design during the first meeting, and they were told about the possible benefits and risks of the research. After familiarization, all the subjects were given a written informed consent form which was prepared according to the Declaration of Helsinki (code DoHOct, 2013).

\section{Results}

Table 1 shows energy expenditure of the participants on a weekday and during the one-hour HY practice. While AEE was $19.16 \%$ of TEE during the weekday, AEE was $68.35 \%$ of TEE during the HY session. The most remarkable finding in Table 1 is that total AEE during one hour of HY practice was equal to $26.01 \%$ of daily AEE. Finally, average PAD increased from 1.53 MET to 2.57 MET throughout the HY practice.

The HRV values obtained at the beginning, during and after the HY practice are displayed in Table 2 . The \%HRmax value of the participants was calculated as 64.97 during the whole HY session. The HRave was higher during HY than all the other ones $(\mathrm{p}<0.01)$, and at HYend than HY5minA $(\mathrm{p}<0.01)$. The SDNN value was lower at HYlOminA than HY5minB, HY, and HYend $(\mathrm{p}<0.01)$. Similarly, SDNN measured at the HY5minA was lower than HY5minB and HY values $(\mathrm{p}<0.05)$. For RMSSD, HY5minB was recorded higher than HY, HYend, HY5minA, and HY10minA values $(\mathrm{p}<0.05)$. SDSD was higher at HY5minB than HY, HYend, and HY5minA ( $<<0.05)$. NN50 and pNN50 were recorded higher at HY5minB than all the other measure- 
ments $(\mathrm{p}<0.05)$. The TRI value was higher at HY5minB than HYend and HY5minA ( $<<0.05)$, and HY was found to be higher than HY5minA $(\mathrm{p}<0.05)$.

The frequency-domain parameters also had significant alterations. LF/HF was noted to be higher at $\mathrm{HY}$ than all the other ones $(p<0.01)$. HF was found to be lower during HY than all the other ones $(\mathrm{p}<0.01)$, and higher at HY5minB than HY, HYend $(\mathrm{p}<0.01)$, and also than HY10minA $(\mathrm{p}<0.05)$. The LF value was lower at HYend than HY5minA ( $<<0.05)$. VLF was found to be higher at HY than HY5minB, HYend and HY5minA ( $<<0.01)$,

Table 1. Energy expenditure of the participants during a weekday and HY practice

\begin{tabular}{|c|c|c|c|}
\hline Parameters & $\begin{array}{c}\text { Energy cost dur- } \\
\text { ing a weekday }\end{array}$ & $\begin{array}{c}\text { Energy cost dur- } \\
\text { ing HY practice }\end{array}$ & $\begin{array}{c}\text { Percentage of } \\
\text { the difference }\end{array}$ \\
\hline TEE (kcal, 0-9 MET) & $2201.40 \pm 137.01$ & $160.50 \pm 9.38$ & 7.29 \\
\hline AEE (kcal, 3-6 MET) & $421.70 \pm 227.00$ & $109.70 \pm 22.12$ & 26.01 \\
\hline NOS & $7246.10 \pm 2114.93$ & $36.50 \pm 12.96$ & 0.50 \\
\hline AMET & $1.53 \pm 0.19$ & $2.57 \pm 0.24$ & 59.53 \\
\hline SD (min) & $407.70 \pm 58.63$ & $\mathrm{~N} / \mathrm{A}$ & $\mathrm{N} / \mathrm{A}$ \\
\hline SE $(\%)$ & $84.20 \pm 6.81$ & $\mathrm{~N} / \mathrm{A}$ & $\mathrm{N} / \mathrm{A}$ \\
\hline $\mathrm{LDD}(\mathrm{min})$ & $483.30 \pm 72.75$ & $\mathrm{~N} / \mathrm{A}$ & $\mathrm{N} / \mathrm{A}$ \\
\hline $\mathrm{SLD}(\mathrm{min})$ & $1327.00 \pm 66.13$ & $32.80 \pm 6.61$ & 2.47 \\
\hline TPAD (min) & $113.60 \pm 64.74$ & $29.20 \pm 6.61$ & 25.70 \\
\hline APAD (min) & $111.90 \pm 64.72$ & $29.20 \pm 6.61$ & 26.10 \\
\hline VPAD (min) & $2.60 \pm 3.72$ & $\mathrm{~N} / \mathrm{A}$ & $\mathrm{N} / \mathrm{A}$ \\
\hline
\end{tabular}

TEE: total energy expenditure; AEE: active energy expenditure; NOS: number of steps; AMET: average MET value; SD: sleep duration; SE: sleep efficiency; LDD: lying down duration; SLD: sedentary life duration; TPAD: total physical activity duration (3-9 MET); APAD: average physical activity duration (3-6 MET); VPAD: vigorous physical activity duration (6-9 MET); N/A: Not applicable. and HY10minA ( $\mathrm{p}<0.05)$. VLF was also lower at HY5minA than HYlOminA $(\mathrm{p}<0.01)$.

\section{Discussion}

The present study tried to achieve two different aims during the HY session. First, energy expenditure was measured and compared with daily energy expenditure. Secondly, HRV changes were recorded to understand the effects of one-hour HY exercise on autonomic heart functions.

According to the results, TEE was $160.50 \pm 9.38 \mathrm{kcal}$ during HY, and this was equal to $7.29 \%$ of the total daily EE. More importantly, AEE was $109.70 \pm 22.12$ during HY activity, and this result was equal to $26.01 \%$ of AEE recorded during the whole day. As a result, the participants' AEE percentage increased from 19.16 to 68.35 . Energy cost of physical activity can be determined with different measurements, and one of the most common ones is MET. MET is defined as $3.5 \mathrm{ml} . \mathrm{kg}^{-1} \cdot \mathrm{min}^{-1}$ oxygen uptake during physical activity, and people are recommended to perform at least 150 minutes of physical activity with the intensity of 3-6 MET per week [21]. The average MET value was found to be $1.53 \pm 0.19$ during a weekday in the current study, and it increased to $2.57 \pm 0.24$ during a one-hour HY session. In this respect, HY has energy cost close to walking at $4.5 \mathrm{~km} / \mathrm{h}$, wind surfing, dancing or non-competitive volleyball playing [27]. Considering that HY lasted 60 minutes and had potential beneficial effects on physical performance parameters such as muscle strength, endurance and flexibility, it could be inferred that $\mathrm{HY}$ is adequate to meet the physical activity requirements even if the MET value is still slightly under 3 MET. When planning the same HY session only for 3 days per week, the MET. min.wk $k^{-1}$ value would be $462.6(2.57 \times 60 \times 3)$, and it would remain in the recommended range (450-750 MET.min.wk $\mathrm{w}^{-1}$ ).

There exist other studies in which EE of a yoga session has been measured. In many of these studies an indirect calorimeter

Table 2. Mean values and comparisons of the HRV parameters derived from 5 minutes before, during, at the end as well as 5 and 10 minutes after the HY session

\begin{tabular}{|c|c|c|c|c|c|c|}
\hline Parameters & HY5minB(a) & HY (b) & HYend (c) & HY5minA (d) & HY10minA (e) & P \\
\hline HRave & $77.40 \pm 10.68$ & $118.84 \pm 7.64$ & $80.80 \pm 8.13$ & $77.20 \pm 7.81$ & $77.70 \pm 8.21$ & $0.000^{* *}$ \\
\hline SDNN & $67.00 \pm 34.36$ & $53.31 \pm 12.01$ & $55.60 \pm 22.24$ & $31.70 \pm 12.68$ & $37.20 \pm 14.88$ & $0.000^{* *}$ \\
\hline RMSSD & $32.70 \pm 16.16$ & $22.13 \pm 10.76$ & $22.00 \pm 9.09$ & $23.10 \pm 12.19$ & $23.40 \pm 9.63$ & $0.026^{*}$ \\
\hline SDSD & $33.60 \pm 16.74$ & $23.15 \pm 10.94$ & $21.80 \pm 9.24$ & $23.10 \pm 12.19$ & $25.20 \pm 9.44$ & $0.040^{*}$ \\
\hline NN50 & $45.70 \pm 44.01$ & $10.76 \pm 6.34$ & $17.10 \pm 20.14$ & $16.80 \pm 29.52$ & $22.60 \pm 29.85$ & $0.044^{*}$ \\
\hline pNN50 & $12.08 \pm 11.90$ & $1.93 \pm 1.20$ & $4.29 \pm 5.04$ & $4.51 \pm 8.05$ & $6.08 \pm 8.03$ & $0.024^{*}$ \\
\hline TRI & $11.38 \pm 4.24$ & $9.77 \pm 1.44$ & $8.98 \pm 2.89$ & $7.90 \pm 2.02$ & $8.88 \pm 2.03$ & $0.016^{*}$ \\
\hline TP & $1112.56 \pm 445.59$ & $1241.48 \pm 466.27$ & $860.59 \pm 445.29$ & $843.71 \pm 701.18$ & $1024.24 \pm 629.40$ & 0.161 \\
\hline LF/HF & $1.62 \pm 1.03$ & $7.41 \pm 2.32$ & $2.09 \pm 1.03$ & $2.26 \pm 1.17$ & $1.71 \pm 0.63$ & $0.000^{* *}$ \\
\hline HF & $317.15 \pm 224.59$ & $49.93 \pm 29.20$ & $159.15 \pm 151.11$ & $213.75 \pm 283.70$ & $215.63 \pm 166.60$ & $0.000^{* *}$ \\
\hline LF & $361.47 \pm 166.29$ & $260.69 \pm 123.12$ & $217.13 \pm 118.52$ & $354.24 \pm 233.02$ & $343.98 \pm 211.22$ & 0.107 \\
\hline VLF & $410.88 \pm 237.39$ & $892.16 \pm 338.23$ & $445.56 \pm 247.72$ & $294.74 \pm 247.34$ & $452.17 \pm 266.52$ & $0.001^{* *}$ \\
\hline ULF & $22.06 \pm 19.82$ & $38.69 \pm 19.65$ & $38.95 \pm 39.41$ & $14.25 \pm 22.35$ & $25.63 \pm 27.08$ & 0.101 \\
\hline
\end{tabular}

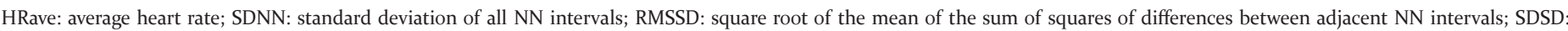

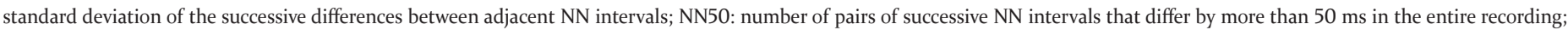

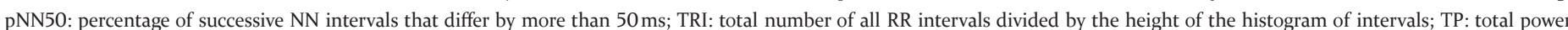

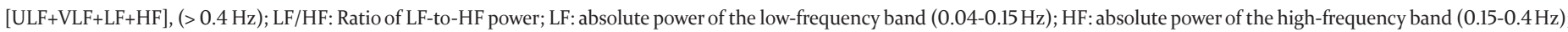

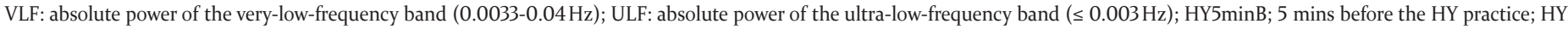
during HY practice; HY end: at the end of the HY practice; HY5minA:5 mins after the HY practice; HY10minA: 10 mins after the HY practice. 
was used, and usually similar caloric responses were found. Pate and Buono [28] compared some physiological responses of novice and experienced yoga practitioners during a 90-min Bikram yoga class, and found that novice practitioners had about 333 kcal, and experienced $423 \mathrm{kcal}$ energy expenditure at the end of the session. The metabolic equivalent of the class was between light to moderate intensity, as it was in the current study. Ce et al. [29] found the energy cost of 6 asanas (each lasting 5 minutes with 5 -minute intervals) about $9.7 \%$ of $\mathrm{VO}_{2}$ max in $10 \mathrm{fe}-$ male professional yoga practitioners, and considered it as light physical activity. In another study, authors stated that professional yoga practitioners need less oxygen consumption when compared to non-yoga practitioners [30]. Mody [31] pointed out that the caloric energy cost in six experienced yoga practitioners was 230 kcal during 30 minutes of Surya Namaskar. Seeing that the total EE was $160.50 \pm 9.38 \mathrm{kcal}$ in the present study, Surya Namaskar seems to generate more energy cost. Therefore, the author suggested a regular Surya Mamaskar program to maintain and/or improve body composition and cardiorespiratory fitness. Ray et al. [32] investigated the energy cost of 20 male experienced yoga practitioners during 37 minutes of HY practice. The mean MET was between 1 and 2, but during the asanas it was $>2$. The present study found it to be 2.57 MET. The results of both studies are very close to each other, and the reason may be the use of the same yoga style. Furthermore, Ray et al. also reported that the intensity of the HY session was between 9.9 and $26.5 \%$ of the participants' $\mathrm{VO}_{2}$ max. Similarly, Hagins et al. [33] found EE to be 2.5 MET after a 52-minute HY session in 20 intermediate to advanced level yoga practitioners, and considered it as low level physical activity. Again, the study on EE in a HY session was performed by Clay et al. [34] in 26 female yoga practitioners, and the MET value was found to be 2.17 after a 30-minute exercise. Another study shows that the energy cost of a yoga session can be enhanced by increasing the speed of yoga [35]. However, in this case, the pranayama phase (breath control, breathing exercises) of a yoga session may be ignored, and it can reduce the effects of long-term yoga exercises on the parasympathetic nervous system [36, 37]. Because of the fact that positive changes gained in cardiac autonomic control, HRV, anxiety, depression and stress occur as a result of slow breathing and the meditation phase $[13,15,38,39,40,41,42]$ during yoga (also known as yogic breathing), neither practitioners nor instructors would prefer to alter it.

In addition to the metabolic parameters such as MET, kcal and oxygen consumption, the preset study also recorded the HRV parameters in order to observe changes in autonomic heart functions. These parameters included HRave, SDNN, RMSSD, SDSD, NN50, pNN50, TRI, TP, HF, LF, VLF, ULF and LF/HF. Even though it was reported in one study that using HR to determine oxygen consumption can cause an overestimation in yoga [43], HR values could still be used to understand heart-based changes and intensity during physical activity. Besides, in accordance with the ACSM, moderate physical activity should be between 64 and 76\% of HRmax [27]. The ratio of HRave to estimated HRmax is $64.97 \%$ during the HY session in the present study. When this result is taken into account together with 2.57 of MET value, it can be said that one-hour HY is equivalent to moderate physical activity. This value was recorded as $72.3 \%$ for the experienced practitioners and $86.4 \%$ for the novices during Birkam yoga in Pate and Buono's study [28]. Ce et al. [29] found the same value as $45 \%$ of the HRmax, and Mody [30] found it to be $80 \%$ during Surya Namaskar yoga. Similar studies were performed in which the subjects participated in a HY session. In these studies, the HRave to HRmax ratio was recorded by Ray et al. [32] as $52.3-54.5 \%$, by Hagins et al. [33] as $49.4 \%$, and by Clay et al. [34] as $56.89 \%$. These results are about $10-20 \%$ lower than the result observed in the present study. It may stem from a longer duration of HY and different poses in the current study.

HRave value was found to be statistically higher during $\mathrm{HY}$ than all the other ones $(p<0.01)$, which means that HY increases heart functions. HRave value was also higher at HYend than at HY5minA ( $\mathrm{p}<0.01)$. This result shows that 5 minutes of the meditation process was not adequate for HR to return to the initial level, and HR could reach the initial level after 10 minutes of recovery ( 5 minutes of the meditation phase and 5 minutes of passive recovery). When examining the time-domain parameters of HRV, significant changes in SDNN ( $<<0.01)$, RMSSD, SDSD, NN50, pNN50, TRI ( $<0.05)$ parameters can be seen. SDNN was found to be lower at HY5minA than HY5minB and HY (< 0.05) and HY10minA than HY5minB, HY, and HYend $(\mathrm{p}<0.01)$. These changes mean that the impairment in SDNN may not be gradual, and 15 minutes of recovery is not sufficient to reach the initial level ( 5 minutes of the meditation phase and 10 minutes of passive recovery). For the RMSSD, NN50, and pNN50, the HY5minB was recorded to be higher than all the other measurements $(\mathrm{p}<0.05)$, which shows again that 15 minutes was not adequate for the full recovery. After a decrease, the SDSD and the TRI values returned to the initial level at HYlOminA. Ten-minute passive recovery was sufficient for both to reach the initial level. In accordance with the time-domain parameters' results, it can be inferred that practicing HY for one hour increases sympathetic activity, and the rise does not occur gradually. Similar changes to the time-domain parameters were also observed in the frequency-domain parameters of HRV. The LF/HF ratio was found to be higher than all the other measurements $(\mathrm{p}<0.01)$. Being one of the substantial indicators of sympathovagal balance, the decrease in LF/HF showed a significant increase in sympathetic activity, and 15 minutes of recovery was not efficient. As an indicator of parasympathetic activity, HF was lower at HY than all the other ones $(\mathrm{p}<0.01)$. Being a marker of increased sympathetic activity, VLF was observed to be higher at HY than HY5minB, HYend, and HY5minA ( $<<0.01)$, and HY10minA ( $\mathrm{p}<0.05)$. There were no significant changes in TP and ULF parameters, and the only change found in LF was the difference between decreased HYend and HY5minA $(\mathrm{p}<0.05)$. Similar to the time-domain parameters of HRV, frequency-domain parameters showed increased sympathetic and decreased parasympathetic activity after a 55-minute HY session, in which the asanas was performed, and neither the 5-minute meditation phase nor the 10-minute passive recovery phase (15 minutes in total) enabled the subjects to return their initial level. Similar changes in HRV values were found by Javorka et al. [44]. Even though they observed increased HRV values during a 30-minute recovery phase in the supine position after 8 minutes of step exercise at a moderate level, the enhancements did not reach the resting value.

There are studies in which changes in HRV parameters were investigated after a meditation process. In one of them, authors compared 20 minutes of meditation (dhyana) with randomly thinking, meditative and non-meditative focusing, and found that meditation increased parasympathetic activity significantly with an increase in HF, NN50, pNN50, and a decrease in LF [45]. Markil et al. [46] investigated the effects of 30-minute yoga nidra relaxation alone and after 60 minutes of a HY session, and observed some positive changes in HRV parameters in both conditions. The HY session was very similar to the one performed in the current study. However, unlike the present study, they found increased vagal activity after the relaxation phase. It 
may stem from a longer resting phase as well as using yoga nidra relaxation instead of supine resting. Santaella et al. [47] compared the 20-minute yoga relaxation (savasana) with resting supine position both in hypertensive patients and normotensive individuals, and reported yoga relaxation to be more effective for enhanced HRV parameters (RR intervals, LFnu, HFnu, and LF/HF). Similar changes in LFnu, HFnu and LF/HF parameters were observed even after 10-minute yoga-based relaxation when compared with supine rest [48]. Travis and Wallace [48], and Travis [49] also stated that transcendental meditation, performed for 10 and 15 minutes respectively, exerts an influence on HRV (increased HF value). The results of the above-mentioned studies show that a single yoga meditation phase enhances HRV. Nevertheless, in the present study, neither the 5-minute meditation phase nor the 10-minute supine rest was effective to reach the initial level in HRV values after implementing moderate-level 55-minute HY. Therefore, it would be beneficial to prolong the meditation time when faster recovery in HRV is desired.

\section{Conclusions}

The energy equivalent of a one-hour HY session was found to be light to moderate when the MET value was taken into account. Besides, according to the \% HRmax value, the intensity of the HY exercise was moderate at the beginning. Although the device used to measure energy expenditure was a metabolic arm holter instead of an indirect calorimeter, having similar caloric responses showed that this method could also be used reliably in such sports. Furthermore, using this kind of armband instead of an indirect calorimeter allows practitioners to move their body much more easily during the activity. In line with the increase in \% HRmax, HRV parameters did increase during the asanas phase, and did not return to initial level after either the meditation or passive recovery phase. These results, observed both in energy expenditure and heart rate variability parameters, prove that this kind of exercise could be used to maintain and/or improve some physical fitness parameters if performed at least 3 days a week. Additionally, compared to supine rest, yoga breathing and yoga-based meditation can be used by athletes and people who exercise to achieve acute positive improvements in HRV. However, the duration may need to be at least 20 minutes after moderate physical activity.

\section{Acknowledgements}

The authors thank all volunteers for their collaboration during the study. This research did not receive any specific grant from funding agencies in the public, commercial, or not-forprofit sectors.

\section{References}

1. Desikachar T.K.V. (1999). The heart of yoga: Developing a personal practice. Vermont: Inner Traditions International.

2. Larson-Meyer D.E. (2016). A systematic review of the energy cost and metabolic intensity of yoga. Medicine and Science in Sport and Exercise 48, 1558-1569. DOI: 10.1249/ MSS.0000000000000922

3. Cramer H., Ward L., Steel A., Lauche R., Dobos G., Zhang Y. (2016). Prevalence, patterns, and predictors of yoga use: results of a U.S. nationally representative survey. American Journal of Preventive Medicine 50(2), 230-235. DOI: 10.1016/j. amepre.2015.07.037
4. Desveaux L., Lee A., Brooks D. (2015). Yoga in the management of chronic disease: a systematic review and meta-analysis. Medical Care 53, 653-661. DOI: 10.1097/ MLR.0000000000000372

5. Wieland L.S., Skoetz N., Pilkington K., Vempati R., D’Adamo C.R., Berman B.M. (2017). Yoga treatment for chronic non-specific low back pain. Cochrane Database of Systematic Reviews 12, 1(1), CD010671. DOI: 10.1002/14651858. CD010671.pub2

6. Uebelacker L.A., Van Noppen D., Tremont C., Bailey G., Abrantesa A., Stein M. (2019). A pilot study assessing acceptability and feasibility of hatha yoga for chronic pain in people receiving opioid agonist therapy for opioid use disorder. Journal of Substance Abuse Treatment 105, 19-27. DOI: 10.1016/j.jsat.2019.07.015

7. Schmalzl L., Powers C., Henjeblom E. (2015). Neurophysiological and neuro cognitive mechanisms underlying the effects of yoga-based practices: towards a comprehensive theoretical framework. Frontiers in Human Neuroscience 235, 1-19. DOI: 10.3389/fnhum.2015.00235

8. Chu P., Gotink R.A., Yeh G.Y., Goldie S.J., Hunink M.G. (2016). The effectiveness of yoga in modifying risk factors for cardiovascular disease and metabolic syndrome: a systematic review and meta-analysis of randomized controlled trials. European Journal of Preventive Cardiology 23(3), 291307. DOI: 10.1177/2047487314562741

9. Cramer H., Lauche R., Anheyer D., Pilkington K., de Manincor M., et al. (2018). Yoga for anxiety: A systematic review and meta-analysis of randomized controlled trials. Depression and Anxiety 35(9), 830-843. DOI: 10.1002/da.22762

10. Papp M.E., Nygren-Bonnier M., Gullstrand L., Wändell P.E., Lindfors P. (2019). A randomized controlled pilot study of the effects of 6-week high intensity hatha yoga protocol on health-related outcomes among students. Journal of Bodywork E Movement Therapies 23(4), 766-772. DOI: 10.1016/j. jbmt.2019.05.013

11. Bower J.E., Woolery A., Sternlieb B., Garet D. (2005). Yoga for cancer patients and survivors. Cancer Control 12, 165-171. DOI: $10.1177 / 107327480501200304$

12. Paprika D., Gingl Z., Rudas L., Zöllei E. (2014). Hemodynamic effects of slow breathing: does the pattern matter beyond rate? Acta Physiologica Hungarica 101(3), 273-281. DOI: 10.1556/APhysiol.101.2014.3.2

13. Vidigal G.A., Tavares B.S., Garner D.M., Porto A.A., Carlos de Abreu L., et al. (2016). Slow breathing influences cardiac autonomic responses to postural maneuver: Slow breathing and HRV. Complementary Therapies Clinical Practice 23, 1420. DOI: 10.1016/j.ctcp.2015.11.005

14. Zou Y., Zhao X., Hou Y.Y., Liu T.W.Q., Wu Q., et al. (2017). Meta-analysis of effects of voluntary slow breathing exercises for control of heart rate and blood pressure in patients with cardiovascular diseases. The American Journal of Cardiology 120(1), 148-153. DOI: 10.1016/j.amjcard.2017.03.247

15. De Couck M., Caers R., Musch L., Fliegauf J., Giangreco A., Gidron Y. (2019). How breathing can help you make better decisions: Two studies on the effects of breathing patterns on heart rate variability and decision-making in business cases. International Journal of Psychophysiology 139, 1-9. DOI: 10.1016/j.ijpsycho.2019.02.011

16. Ikai S., Uchida H., Mizuno Y., Tani H., Nagaoka M., et al. (2017). Effects of chair yoga therapy on physical fitness in patients with psychiatric disorders: A 12-week single-blind randomized controlled trial. Journal of Psychiatric Research 94, 194-201. DOI: 10.1016/j.jpsychires.2017.07.015

17. Beazley D., Patel S., Davis B., Vinson S., Bolgla L. (2017). Trunk and hip muscle activation during yoga poses: Im- 
plications for physical therapy practice. Complementary Therapies in Clinical Practice 29, 130-135. DOI: 10.1016/j. ctcp.2017.09.009

18. Bishop S.R., Lau M., Shapiro S., Carlson L., Anderson N.D., et al. (2014). Mindfulness: A proposed operational definition. Clinical Psychology: Science and Practice 11, 230-241. DOI: 10.1093/clipsy.bph077

19. Park C.L., Elwy A.R., Maiya M., Sarkin A.J., Riley K.E., et al. The essential properties of yoga questionnaire (EPYQ): Psychometric properties. International Journal of Yoga Therapy 28(1), 23-38. DOI: 10.17761/2018-00016R2

20.Zou L., Yeung A., Li C., Wei G.X., Chen K.W., et al. (2018). Effects of meditative movements on major depressive disorder: A systematic review and meta-analysis of randomized controlled trials. Journal of Clinical Medicine 7(8), 195. DOI: 10.3390/jcm7080195

21. Haskell W.L., Lee I.M., Pate R.R., Powell K.E., Blair S.E., et al. (2007). Physical activity and public health: updated recommendation for adults from the American College of Sports Medicine and the American Heart Association. Medicine and Science in Sports and Exercise 39(8), 1423-1434. DOI: 10.1161/CIRCULATION.107.185649

22. Myllymaki T., Rusko H., Syvaoja H., Juuti T., Kinnunen M.L., Kyrolainen H. (2012). Effects of exercise intensity and duration on nocturnal heart rate variability and sleep quality. European Journal of Applied Physiology 112(3), 801-809. DOI: $10.1007 /$ s00421-011-2034-9

23. Pascoe M.C., Bauer I.E. (2015). A systematic review of randomized control trials on the effects of yoga on stress measures and mood. Journal of Psychiatric Research 68, 270-282. DOI: 10.1016/j.jpsychires.2015.07.013

24. Vizcaino M., Stover E. (2016). The effect of yoga practice on glycemic control and other health parameters in Type 2 diabetes mellitus patients: A systematic review and meta-analysis. Complementary Therapies in Medicine 28, 57-66. DOI: 10.1016/j.ctim.2016.06.007

25. Gellish R.L., Goslin B.R., Olson R.E., McDonald A., Russi G.D., Moudgil V.K. (2007). Longitudinal modeling of the relationship between age and maximal heart rate. Medicine and Science in Sports and Exercise 39(5), 822-829. DOI: 10.1097/mss.0b013e31803349c6

26. Aylin Y., Giray K., Ebru A., Tokgozoglu L., Oto A. (2002). Effects of menstrual cycle on cardiac autonomic innervations as assessed by heart rate variability. Annals of Noninvasive Electrocardiology 7(1), 60-63. DOI: 10.1111/j.1542-474x.2001. tb00140.x

27. Riebe D., Ehrman J.K., Liguori G., Magal M. (2018). ACSM's Guidelines for Exercise Testing and Prescription. Philadelphia, PA: Wolters Kluwer

28. Pat J., Buono M. (2014). The physiological responses to Bikram yoga in novice and experienced practitioners. Alternative Therapies in Health and Medicine 20(4), 12-19.

29. Cè E., Maggioni M.A., Boniello S., Veicsteinas A., Merati G. (2015). Anthropometric and physiologic profiles of female professional yoga practitioners and energy expenditure during asanas execution. Journal of Sports Medicine and Physical Fitness 55(1-2), 51-57.

30. Tyagi A., Cohen M., Reece J., Telles S. (2014). An explorative study of metabolic responses to mental stress and yoga practices in yoga practitioners, non-yoga practitioners and individuals with metabolic syndrome. BMC Complementary and Alternative Medicine 14, 445. DOI: 10.1186/1472-6882$14-445$
31. Mody B.S. (2011). Acute effects of Surya Namaskar on the cardiovascular \& metabolic system. Journal of Bodywork and Movement Therapies 15(3), 343-347. DOI: 10.1016/j. jbmt.2010.05.001

32. Ray U..S., Pathak A., Tomer O.S. (2011). Hatha yoga practices: energy expenditure, respiratory changes and intensity of exercise. Evidence-Based Complementary and Alternative Medicine, ID 241294. DOI: 10.1093/ecam/neq046

33. Hagins M., Moore W., Rundle A. (2007). Does practicing Hatha yoga satisfy recommendations for intensity of physical activity which improves and maintains health and cardiovascular fitness? BMC Complementary and Alternative Medicine 7(1), 40. DOI: 10.1186/1472-6882-7-40

34. Clay C.C., Lloyd L.K., Walker J.L., Sharp K.R., Pankey R.B. (2005). The metabolic cost of Hatha yoga. Journal of Strength and Conditioning Research 19(3), 604-610.

35. Potiaumpai M., Martins M.C.M., Rodriguez R., Mooney K., Signorile J.F. (2016). Differences in energy expenditure during high-speed versus standard-speed yoga: A randomized sequence crossover trial. Complementary Therapies in Medicine 29, 169-174. DOI: 10.1016/j.ctim.2016.10.002

36. Telles S., Sharma S., Gupta R., Bhardwaj A., Balkrishna A. (2016). Heart rate variability in chronic low back pain patients randomized to yoga or standard care. BMC Complementary and Alternative Medicine 16(1), 279. DOI: 10.1186/ s12906-016-1271-1

37. Zou L., Sasaki J.E., Wei G.X., Huang T., Yeung A.S., et al. (2018). Effects of Mind-Body Exercises (Tai Chi/Yoga) on Heart Rate Variability Parameters and Perceived Stress: A Systematic Review with Meta-Analysis of Randomized Controlled Trials. Journal of Clinical Medicine 7(11), 404. DOI: 10.3390/jcm7110404

38. Brown R.P., Gerbarg P.L. (2005). Sudarshan Kriya Yogic breathing in the treatment of stress, anxiety, and depression. Part II - Clinical Applications and Guidelines. Journal of Alternative and Complementary Medicine 11(4), 711-717. DOI: 10.1089/acm.2005.11.711

39. Doria S., de Vuono A., Sanlorenzo R., Irtelli F., Mencacci C. (2015). Anti-anxiety efficacy of Sudarshan Kriya Yoga in general anxiety disorder: a multicomponent, yoga based, breath intervention program for patients suffering from generalized anxiety disorder with or without comorbidities. Journal of Affective Disorders 184, 310-317. DOI: 10.1016/j. jad.2015.06.011

40.Tyagi A., Cohen M. (2016). Yoga and heart rate variability: A comprehensive review of the literature. International Journal of Yoga 9, 97-113. DOI: 10.4103/0973-6131.183712

41. Toschi-Dias E., Tobaldini E., Solbiati M., Costantino G., Sanlorenzo R., et al. (2017). Sudarshan Kriya Yoga improves cardiac autonomic control in patients with anxiety-depression disorders. Journal of Affective Disorders 214, 74-80. DOI: 10.1016/j.jad.2017.03.017

42. Woody A., Figueroa W.S., Benencia F., Zoccola P.M. (2017). Stress-induced parasympathetic control and its association with inflammatory reactivity. Psychosomatic Medicine 79, 306-310. DOI: 10.1097/PSY.0000000000000426

43. Carroll J., BlansitA., Otto R.M., Wygand J.W. (2003). The metabolic requirements of Vinyasa Yoga. Medicine and Science in Sports and Exercise 35, 155. DOI: 10.1123/jmpb.2017-0010

44. Javorka M., Zila I., Balhárek T., Javorka K. (2002). Heart rate recovery after exercise: relations to heart rate variability and complexity. Brazilian Journal of Medical and Biological Research 35(8), 991-1000. DOI: 10.1590/s0100$-879 \times 2002000800018$ 
45. Telles S., Raghavendra B.R., Naveen K.V., Manjunath N.K., Kumar S., Subramanya P. (2013). Changes in autonomic variables following two meditative states described in yoga texts. Journal of Alternative and Complementary Medicine 19, 35-42. DOI: $10.1089 / \mathrm{acm} .2011 .0282$

46. Markil N., Whitehurst M., Jacobs P.L., Zoeller R.F. (2012). Yoga Nidra relaxation increases heart rate variability and is unaffected by a prior bout of Hatha Yoga. Journal of Alternative and Complementary Medicine 18(10), 953-958. DOI: 10.1089/acm.2011.0331

47. Santaella D.F., Lorenzi-Filho G., Rodrigues M.R., Tinucci T., Malinauskas A.P., et al. (2014). Yoga relaxation (Savasana) decreases cardiac sympathovagal balance in hypertensive patients. Medical Express 1(5), 233-238. DOI: 10.5935/MedicalExpress.2014.05.04

48. Travis F., Wallace R.K. (1999). Autonomic and EEG patterns during eyes-closed rest and transcendental meditation (TM) practice: The basis for a neural model of TM practice. Consciousness and Cognition 8, 302-318. DOI: 10.1006/ ccog.1999.0403

49. Travis F. (2001). Autonomic and EEG patterns distinguish transcending from other experiences during transcendental meditation practice. International Journal of Psychophysiology 42, 1-9. DOI: 10.1016/S0167-8760(01)00143-X

Submitted: November 6, 2020

Accepted: December 31, 2020 